\title{
The Frequency and Associated Risk Factors for Dementia in Patients With Parkinson's Disease
}

\author{
Karen Marder, MD, MPH; Ming-Xin Tang, PhD; Lucien Cote, MD; Yaakov Stern, PhD; Richard Mayeux, MD, MSE
}

Objectives: To estimate the frequency and determine the risk factors for incident dementia in communitydwelling patients with Parkinson's disease (PD) and in control subjects.

Design: Prospective cohort study. During a 3.5-year period, 140 patients with idiopathic PD without evidence of dementia and 572 nondemented control subjects were identified in the community of Washington Heights-Inwood in New York, NY. All subjects underwent neurological and neuropsychological evaluations and follow-up examinations.

Results: Twenty-seven patients with PD (19.2\%) became demented throughout 2 years, as compared with 87 (15.2\%) of the control subjects. The relative risk (RR) for the development of dementia with PD was 1.7 (95\% confidence interval $[\mathrm{CI}], 1.1$ to 2.7 ) after adjusting for age, education, and gender. Predictive features of incident dementia were an extrapyramidal score greater than 25 (RR, $3.56 ; 95 \% \mathrm{CI}$, 1.4 to 8.9 ) and a Hamilton Depression Rating Scale score greater than 10 (RR, $3.55 ; 95 \% \mathrm{Cl}, 1.6$ to 7.9 ).

Conclusion: Patients with PD, especially those with severe extrapyramidal signs, have almost twice the risk for the development of dementia than do communitydwelling control subjects.

(Arch Neurol. 1995;52:695-701)
From the Gertrude H. Sergievsky Center

(Drs Marder, Tang, Stern, and Mayeux), the Departments of Neurology (Drs Marder, Tang, Cote, Stern, and Mayeux) and Psychiatry (Drs Stern and Mayeux); College of Physicians and Surgeons; the Center for Alzheimer's Disease Research in the City of New York (Drs Marder, Tang, Cote, Stern, and Mayeux), and the Division of Epidemiology, School of Public Health,

Columbia University

(Dr Mayeux), New York, NY.

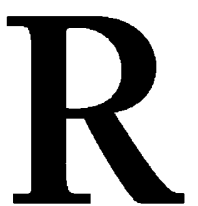

ISK FACTORS that are associated with dementia in patients with Parkinson's disease (PD) include older age at the onset of motor manifestations, severity of the extrapyramidal syndrome (particularly bradykinesia), a family history of dementia, psychological stress (eg, Holocaust), depression, hypertension, and low socioeconomic or low educational attainment. ${ }^{1-3}$ Most studies of risk factors for dementia in patients with PD have been retrospective, although four longitudinal studies have examined the predictive value of baseline characteristics of nondemented patients with PD. ${ }^{4-7}$

In our previous cross-sectional studies of patients with $\mathrm{PD}$, we hypothesized that the factors associated with dementia, ie, age at onset of motor manifestations, severity of extrapyramidal signs, and depressive symptoms, if present at baseline examination in nondemented patients with PD, would predict the development of dementia.

We examined the risk for the development of dementia in patients with PD as compared with that in nondemented control subjects with no known neurological disease, which was ascertained in a similar manner, from the same community.

\section{RESULTS}

Between April 1, 1988 and December 31, 1991, we evaluated 353 patients with possible PD. Two hundred seventy-nine patients met research criteria for PD. ${ }^{8-10}$ An additional 37 patients had essential tremor, and 37 had other disorders, but not PD.

Of the 279 patients who met research criteria for PD, 104 (37.3\%) were demented at their first evaluation and were not included in the analysis.

Of 175 nondemented patients with $\mathrm{PD}, 35$ were seen only once and were not included in the proportional hazards analysis. Fourteen of the 35 patients, who were seen only once, died within the year prior to their first follow-up; 12 were not yet due for their follow-up; and nine could not be found despite repeated attempts to contact them.

\section{See Methods on next page}




\section{METHODS}

\section{SUBJECTS AND SETTING}

All patients with PD and control subjects were participants in a study of degenerative disease in elderly persons who were living in the Washington Heights-Inwood section of northern Manhattan (New York, NY). A registry of cases for several diseases, including $\mathrm{PD}$, was developed from a number of sources: regional hospitals (including inpatient and outpatient services), private practitioners in the community, health maintenance organizations, senior citizens centers, federal and state health agencies, and newspaper and radio announcements. Neurology clinic records at Harlem Hospital, New York, NY, were reviewed for cases of PD, and two neurologists at the Allen Pavilion, a satellite hospital at the tip of Manhattan, referred all cases of PD to the registry. Records of patients at the regional Health Insurance Providers of New York (NY) were screened for PD. In addition, the Health Resources Association, New York, allowed us to review all patients' records in the area who had home health aides.

\section{CRITERIA FOR IDIOPATHIC PD}

Idiopathic PD was defined by clinical and research criteria. ${ }^{8-10}$ We excluded patients with secondary or symptomatic parkinsonism, ie, parkinsonism that resulted from treatment with phenothiazines, methyldopa (alphamethyldopa), reserpine, or metoclopramide hydrochloride. Patients with clinical presentations that were suggestive of progressive supranuclear palsy, essential tremor, ShyDrager syndrome, presumed striatonigral degeneration, and olivopontocerebellar atrophy were excluded. Patients who by history from an informant experienced cognitive impairment that was sufficient to meet criteria for dementia prior to the development of the motor manifestations of PD without any atypical features were considered in the majority of cases to have the "extrapyramidal form" of Alzheimer's disease." They were excluded in an effort to include only patients with idiopathic PD who were nondemented at their baseline visit.

\section{CRITERIA FOR CONTROL SUBJECTS}

Control subjects were volunteers who were living in the same geographical area in northern Manhattan. Control subjects were also referred by community-based health providers, and they were recruited from senior citizens centers and housing, nursing homes that were serving local residents, and a state agency list of home care recipients.
Requirements included (1) being nondemented at baseline examination based on the neuropsychological paradigm and (2) having no evidence of any neurodegenerative disease, such as PD or stroke. Depression was not an exclusion in the group of control subjects. All control subjects were seen by a neurologist and were older than 60 years when they were recruited.

\section{NEUROLOGICAL ASSESSMENT}

Extrapyramidal signs were rated by using the Unified Parkinson's Disease Rating Scale (UPDRS). ${ }^{12}$ Reliability of the assessment of extrapyramidal signs by the three raters (K.M., L.C., and R.M.) for the presence of resting tremor, rigidity, rapid-alternating movements, bradykinesia, and gait was previously demonstrated to be excellent in patients with PD. ${ }^{13}$

An extrapyramidal sign score was calculated by summing the scores on the individual items that were rated on the motor portion of the UPDRS. All items were rated from a score of 0 to 4 , with 0 being normal and 4 indicating severe impairment. Individual limbs, the face, and the neck were assessed separately for tremor at rest and rigidity. Action tremor was rated only in the arms. Other items that were included were speech, facial expression, opening/ closing the hands, pronation/supination of the hands, leg agility, arising from a chair, posture, postural stability, gait, and bradykinesia.

A shortened version of the UPDRS was used to rate extrapyramidal signs in control subjects. Assessment of resting tremor, rigidity, speech, facial expression, posture, and bradykinesia was done by using the items from the UPDRS.

\section{NEUROPSYCHOLOGICAL TEST BATTERY}

Subjects were considered demented if they met the research criteria established for the diagnosis in this population, based on the criteria according to the Diagnostic and Statistical Manual of Mental Disorders, Revised Third Edition (DSM-III-R), ${ }^{14}$ and if they or an informant reported functional difficulties that could be attributed to cognitive rather than physical disability. The neuropsychological battery was used to operationalize the DSM-III-R criteria for cognitive impairment. Details of the paradigm have been reported previously. ${ }^{15}$

\section{OTHER ASSESSMENTS}

Demographical information that was collected included age and education. Duration of PD was calculated based on either the patient's age or the date at which motor signs began (age at onset of PD) until the baseline examination.

All subjects were asked about current medication use
The 35 patients who underwent only a baseline examination and were not included in the Cox proportional hazards model were significantly older (age, 74.1 years) $(P=.05)$ than were the 140 patients who were followed up (age, 71.1 years) but were not significantly different in age at onset of $\mathrm{PD}$, duration of $\mathrm{PD}$, gender, education, mean EPS, or mean HDRS score. A significantly higher proportion of those with only a baseline visit (35.5\%) as compared with those who were followed up $(19.1 \%)$ had HDRS scores of 11 or higher. During the study period, $40 \%$ of those with only a baseline visit died, as compared with $11.4 \%$ of those who were followed up.

An additional 40 patients had only 1 year of followup. At the 1-year follow-up visit, 29 remained nondemented and 11 had become demented. Six of the 40 patients died prior to their 2-year visit, 30 were not yet due for their second annual visit, and four were unavailable for follow-up.

One hundred patients had a 2-year follow-up 
and medical history, including cardiovascular disease (eg, stroke) and head injury with loss of consciousness. Use of dopamine agonists, anticholinergics, antidepressants, and anxiolytics was recorded, but response to medication and dose were not systematically noted. Informants were able to corroborate historical data. Signs on neurological examination, even in the absence of a known history of stroke or computed tomographic or magnetic resonance imaging evidence of stroke, were considered indicative of stroke.

To assess activities of daily living, we used the Activities of Daily Living (ADL) Scale of Schwab and England. ${ }^{16}$ If an informant was available, information from both the patient and the informant was used to rate the patient's condition.

Depressive symptoms were rated by using the standardized semistructured interview for the Hamilton Depression Rating Scale (HDRS). ${ }^{17}$ This scale was not used to make a DSM-III-R diagnosis of major depression, but rather to enumerate depressive symptoms. A total score was calculated by summing the scores on the 17 individual items.

\section{DATA ANALYSIS}

\section{Patients With PD}

To determine the baseline characteristics associated with the development of dementia, patients with PD were divided into two groups based on their dementia status after a maximum of 2 years of follow-up. Patients and control subjects who were seen only once were not included in the analysis.

The subjects who remained nondemented during their observation period were compared with those who had a new onset of dementia on many clinical variables that were related to the study hypothesis. These variables were collected at their baseline visit and were compared by using $\chi^{2}$ tests or $t$ tests. Those variables with a significance level of $<.10$ were included in a Cox proportional hazards model to predict incident dementia. Median values were substituted for continuous variables, with the exception of the HDRS; the HDRS scores were evaluated in three separate ways. The median HDRS score was used in the Cox model; however, because the median score of 5 was too low to be representative of either dysthymia or major depression, we also chose an HDRS score of greater than 10, which we believed was representative of significant depressive symptoms-although not indicative of meeting DSM-III-R criteria for major depression. In a longitudinal study of patients with PD, Starkstein et $\mathrm{l}^{6}$ found that patients who met DSMIII-R criteria for major depression had a mean HDRS score of 15.4 (SD, 3.4), while patients who met criteria for dysthymia had a mean HDRS score of $8.4(\mathrm{SD}, 2.3)$ and those with no depression had a mean HDRS score of $2.2(\mathrm{SD}, 1.6)$.
In a sample of 36 patients from our registry in the Washington Heights-Inwood community that included 13 patients with PD, who were evaluated with both the HDRS and the Structured Clinical Interview for DSM-III-R, an HDRS score greater than or equal to 10 had $50 \%$ sensitivity and $83 \%$ specificity for a DSM-III-R diagnosis of major depression but only a $38 \%$ positive predictive value. The negative predictive value of an HDRS score of 10 or greater was $89 \%$. In the sample of 36 patients, the most sensitive predictor of major depression was the mood item of the HDRS (83\%), even when it was dichotomized as absent (score, 0 ) or 1 or greater. Therefore, we also included the mood item in the Cox model; this mood item was dichotomized as present or absent to predict incident dementia.

An ADL Scale score of $70 \%$, which represented the median score, indicated the first level at which a subject was not completely independent. In this sample, the ADL Scale scores were highly correlated with those of both the Barthel Index ${ }^{18}$ and the Blessed Functional Activity Scale (Part I). ${ }^{19}$

Data from those patients with fewer than 2 years of follow-up-either because of recent study entry or unavailability for follow-up, were censored. Study end points were either the development of dementia, death, or being alive and nondemented at the end of 2 years of follow-up.

Determination of the Relative Risk (RR) of Dementia in PD

The incidence of dementia among nondemented patients with PD was compared with the incidence of dementia among control subjects throughout the follow-up period. Cox proportional hazard models were used to calculate the RR of dementia, when data from nondemented patients with PD and control subjects were compared. The $\chi^{2}$ tests or $t$ tests were used to compare the demographical characteristics of the group of patients with PD and the group of control subjects, and appropriate covariates were included in the model.

In a separate analysis, using only the items on the UPDRS that were common to both the evaluations of patients with PD and the control subjects, the patients with PD were split into two groups (those with high extrapyramidal scores [EPSs] and those with low EPSs) based on the median extrapyramidal sign score. Control subjects were divided solely on the basis of the presence or absence of extrapyramidal signs because EPSs were so infrequent and mild in the group of control subjects. None of the control subjects met research criteria for PD. ${ }^{8-10}$ The control subjects without extrapyramidal signs were considered a reference group, and the three other groups (those with PD with a high EPS, those with PD with a low EPS, and control subjects with extrapyramidal signs) were included in a Cox model, adjusting for age, education, and gender. visit, at which time 84 were nondemented and 16 were demented. A total of 27 patients became demented during the 2-year period, resulting in an incidence rate of $112.5 / 1000$ person-years of observation in this cohort.

Baseline characteristics of the 140 patients who were included in the Cox model are presented in Table 1. Patients who did not become demented were significantly younger at the time that their motor signs of PD began than those with incident dementia. Dis- ease duration, gender, and education did not differ among those who remained nondemented and those with incident dementia.

Thirty-nine of the 140 patients who were not demented at baseline had cognitive impairment that was insufficient to meet criteria for dementia. Sixteen of these 39 patients were among the 27 patients who eventually became demented.

The frequency of head injury and stroke did not significantly differ in those patients who did and did not 


\begin{tabular}{|c|c|c|c|}
\hline \multirow[b]{2}{*}{ Charadteristic } & \multicolumn{2}{|c|}{ Patients } & \multirow[b]{2}{*}{ Significance } \\
\hline & $\begin{array}{l}\text { Nondemented } \\
(n=113)\end{array}$ & $\begin{array}{l}\text { Demented } \\
\text { (n-27) }\end{array}$ & \\
\hline Age, $y$ & $69.9(10.4)$ & $763(6.9)$ & .001 \\
\hline Age at onset of PD, y & $63.9(12.5)$ & $69.9(9.7)$ & 01 \\
\hline Duration of PD, $y$ & $6.5(7.3)$ & $6.8(7.2)$ & NS \\
\hline \multicolumn{4}{|l|}{ Sex, $\%$} \\
\hline$F$ & 55 & 44 & NS \\
\hline$M$ & 45 & 56 & NS \\
\hline Duration of education, y & $11,1(4.9)$ & $8.9(4.3)$ & NS \\
\hline \multicolumn{4}{|l|}{ Score } \\
\hline EPS & $23.7(11.8)$ & $35.5(13.8)$ & .001 \\
\hline HDRS. & $5.3(4.7)$ & $8.9(6.5)$ & .001 \\
\hline
\end{tabular}

*PD indicates Parkinson's disease; NS, not significant; EPS, extrapyramidal score; and HDRS, Hamilton Depression Rating Scale. Data are given as means (SDs). Analysis was performed with tor $\chi^{2}$ tests.

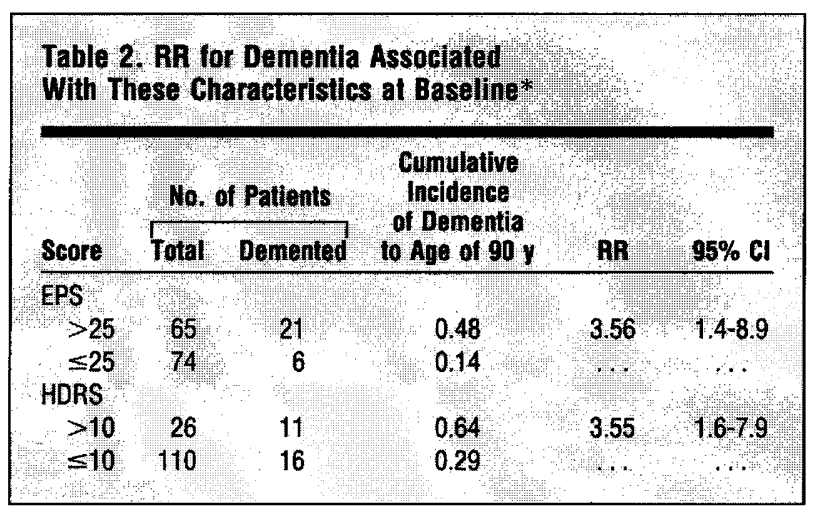

* $R R$ indicates relative risk; $C l$, confidence interval; EPS, extrapyramidal score; and HDRS, Hamilton Depression Rating Scale.

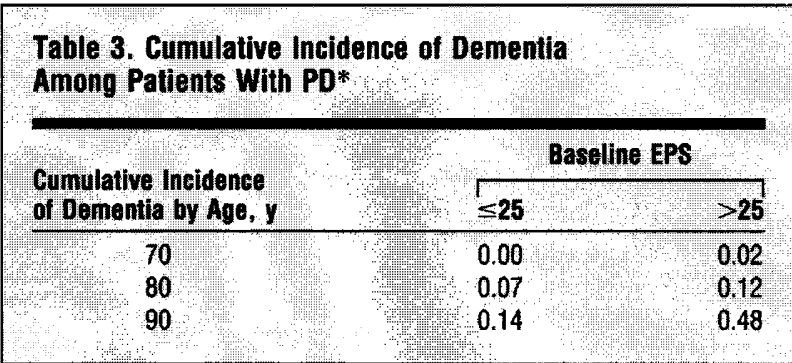

*PD indicates Parkinson's disease; EPS, extrapyramidal score.

become demented. Seventy-six percent of the 140 patients were receiving at least one medication for the treatment of PD at their baseline evaluation, when all were nondemented. The percentage of patients who were receiving levodopa (nondemented, $62.8 \%$; demented, $63 \%$ ), anticholinergics (nondemented, 14.2\%; demented, $18.5 \%$ ), and dopamine agonists (nondemented, $28.3 \%$; demented, $29.6 \%$ ) did not differ at the baseline examination between those who remained nondemented and those who became demented.

All 140 nondemented patients were forward loaded into the Cox proportional hazards model. The following covariates were entered into the Cox proportional hazards model: gender, education, median EPS, median ADL
Scale score, and an HDRS score greater than 10. Stratification of the entire group by age at the time of baseline evaluation ( $>72$ or $\leq 72$ years) was performed. The RRs of dementia associated with these risk factors are shown in Table 2.

An EPS greater than 25 and an HDRS score greater than 10 were independently and significantly associated with dementia in this cohort. Education, gender, ADL Scale score, and age at onset did not contribute significantly to the model.

Cumulative incidence of dementia increased with advancing age in patients with severe EPSs (Table $\mathbf{3}$ ). The cumulative incidence of dementia by the age of 90 years in patients with a baseline total EPS greater than 25 was $48 \%$, as compared with $14 \%$ in those with a total EPS less than or equal to 25 (Tables 2 and 3). The cumulative incidence of dementia by the age of 90 years in patients with a baseline HDRS score greater than 10 was $64 \%$, as compared with $29 \%$ in those with an HDRS score less than or equal to 10 (Table 2).

\section{DEPRESSION}

Depression was also evaluated by substituting the median HDRS score of 5 instead of an HDRS score greater than 10 in the Cox model. An EPS greater than 25 remained significant (RR, 3.4), but depression, measured by the median HDRS score, was not $(P<.09)$. When the presence or absence of depressed mood was substituted for the median HDRS score in the Cox model with the identical covariates, it too was not an independent predictor of dementia, although it approached statistical significance $(P<.07)$.

\section{RISK OF DEMENTIA IN PATIENTS WITH PD AS COMPARED WITH THAT IN CONTROL SUBJECTS}

Five hundred seventy-two control subjects were nondemented at their baseline visit and had at least one follow-up visit. Eighty-seven patients became demented during the 2-year period. Patients with PD were significantly younger than control subjects (mean age of patients with PD, 71.1 years; mean age of control subjects, 73.9 years), better educated (duration of education of patients with $\mathrm{PD}, 10.7$ years; duration of education of control subjects, 9.6 years), and more frequently men (patients with PD, $47.1 \%$; control subjects, $26.9 \%$ ). Therefore, in addition to group (patients with PD or control subjects), gender and education (duration, $>8$ or $\leq 8$ years) were included as covariates in the model to predict incident dementia. The Cox model was stratified by the median age ( $>72$ or $\leq 72$ years).

When gender, education, and group membership were included in the model, group membership (control subjects or patients with PD) and education were significantly associated with the incidence of dementia. A patient with PD was almost twice as likely to become demented than someone without PD (RR, $1.7 ; 95 \%$ confidence interval $[\mathrm{CI}], 1.1$ to 2.7 ), even after adjusting for age, education, and gender. 
Depression was not an exclusion in the group of control subjects. The mean HDRS score in the control subjects was 4.5, and the median HDRS score was 3.0. Control subjects were significantly less likely to be taking antidepressants at baseline $(2.5 \%)$ than were patients with PD (9.7\%) and were also less likely to be taking anxiolytics (3.6\%), compared with $13 \%$ of the patients with PD. In the group of control subjects, the total HDRS score was correlated with the total EPS $(r=.15, P<.02)$. No correlation was seen between the total HDRS score and the total EPS in patients with $P D$. Therefore, it is unlikely that the presence of depression in the group of patients with PD accounted for their higher EPSs.

In the second analysis, the EPSs were examined in patients with PD and control subjects to determine their contribution to the risk of dementia. For this analysis, 349 control subjects and 139 patients with PD had EPSs recorded. Only the EPS items that were common to the evaluations of both the patients with PD and control subjects were included. By using the abbreviated EPS form, patients with PD were stratified into high and low EPSs by the median EPS, which was 14. The EPSs were classified as present or absent in control subjects because the median score was 0 , and the mean EPS for control subjects was 1.6. One hundred eighty-one control subjects had no extrapyramidal signs and 168 had extrapyramidal signs. Only the group of patients with PD who had high EPSs was associated with a significant risk of incident dementia (RR, 2.3; 95\% CI, 1.2 to 4.3 ), while neither of the other groups (patients with PD with low EPSs or control subjects with EPSs) were at an increased risk for the development of dementia as compared with that for control subjects with no extrapyramidal signs (adjusting for education).

Richards et $\mathrm{al}^{20}$ reported that subtle EPSs have been shown to predict the development of dementia in elderly individuals without dementia, independent of age, education, or gender. Therefore, the analysis was repeated with the group of control subjects who were stratified into a low-EPS group (no EPS or one EPS) and a highEPS group that represented the presence of at least two EPSs at baseline. The patients with PD who had high EPSs were again twice as likely as the control subjects with low EPSs to experience dementia (RR, 2.3). Control subjects with high EPSs had an RR for the development of dementia of 1.5 (95\% CI, 0.95 to 2.5 ) that approached but did not reach statistical significance $(P<.07)$, as compared with that in control subjects with low extrapyramidal signs.

\section{COMMENT}

We found that having severe extrapyramidal signs was the best predictor for the development of dementia, independent of age in patients with PD. Age at onset of the motor signs of PD was not a significant independent predictor of incident dementia in this cohort, although the stratification of age ( $\leq 67$ or $>67$ years) would not be considered a traditional cutpoint for young- and olderonset PD. We intentionally tried to recruit a cohort of patients who were older than 60 years at their baseline visit.

Although more severe depressive symptoms were associated with an independent risk for the development of dementia, it is unclear from this analysis whether depressive symptoms are a true risk factor or an antecedent for the development of dementia since the high HDRS scores $(>10)$ were specific but not sensitive indicators of DSM-III-R criteria for major depression. Moreover, by substituting the mood item from the HDRS, which was the most sensitive and specific indicator of major depression, or the median HDRS score in the proportional hazards model, depression was not an independent risk factor for dementia, although a high EPS remained so.

To our knowledge, this is the first study to examine the incidence of dementia in patients with PD as compared with that in community-dwelling elderly persons without neurological impairment. We found that having PD increases the risk of dementia almost twofold after adjusting for age, education, and gender. In a similar analysis, an increased risk of dementia was seen only in the patients with PD who had the most severe extrapyramidal signs, and not in the patients with PD who had lower EPSs or in control subjects with extrapyramidal signs when control subjects without extrapyramidal signs were considered as a reference.

\section{EXTRAPYRAMIDAL SCORE}

$\mathrm{We}^{1}$ and other investigators ${ }^{21}$ have previously reported that in patients with PD, the severity of the EPS is associated with dementia. Dopamine deficiency might underlie both cognitive and motor manifestations. Dementia in patients with PD has been linked to neuronal loss in the medial substantia nigra, which projects to the caudate nucleus and frontal association area, ${ }^{22}$ while the classic motor manifestations of PD are associated with a loss of dopaminergic neurons in the lateral substantia nigra that project to the putamen and then to the premotor areas. The relationship between the severity of the EPS and dementia might represent a parallel decline of these two dopaminergic pathways. Of course, other neurotransmitter systems may be contributing to the development of dementia. Several single-photon emission computed tomographic studies have shown significantly lower temporoparietal perfusion in demented compared with nondemented patients. ${ }^{23-25}$ Also, pathological changes that have been reported in patients with PD who had dementia include those associated with Alzheimer's disease and Lewy body disease.

\section{DEPRESSION}

Depression has been reported in $4 \%$ to $70 \%$ of patients with PD, depending on the patient selection criteria, assessment techniques, and diagnostic criteria for depression. A majority of recent studies that have used standardized rating scales and formal criteria have reported frequencies of $40 \%{ }^{26}$ 
Using the Present State Examination, patients with major depression at baseline were more likely to show significant cognitive and functional decline than were patients with minor or no depression, when they were assessed at 1 year later, regardless of whether the depression had resolved at follow-up. ${ }^{6}$ Widespread pathological changes have been found in the dopaminergic ventral tegmental area in patients with PD who had depression and cognitive impairment. ${ }^{27}$ This area has been linked to reward and reinforcement in animals. Disruption might cause apathy or lack of motivation-symptoms that are often seen in depression. A further link between depression and cognitive impairment in patients with $\mathrm{PD}$ was reported by Mayberg et al. ${ }^{28}$ When patients with PD who had depression were matched to nondepressed patients with PD by age, disease duration, and stage of illness, positron emission tomographic studies revealed a significantly lower metabolic activity in the head of the caudate nucleus and in the orbital-inferior frontal cortex in the depressed patients. ${ }^{28}$ Degeneration of the mesocorticolimbic dopaminergic system was suggested, but in addition, a loss of cortical outflow from this region to the dorsal raphe might explain a secondary serotoninergic deficiency. Serotonin metabolites have been associated with depression in patients with $\mathrm{PD},{ }^{29-30}$ and patients with both depression and dementia have the lowest levels of 5-hydroxyindoleacetic acid. ${ }^{31}$ Depressive symptoms may be associated with dementia because of common dopaminergic and serotoninergic deficits. Clearly, there is a possibility of an anatomical and neurochemical substrate in PD that makes patients vulnerable to both conditions.

\section{RISK OF PD AS COMPARED WITH THAT IN CONTROL SUBJECTS}

We found that nondemented patients with PD were almost twice as likely to develop dementia than were nondemented control subjects from the community. This estimate is probably conservative. The incidence rate of dementia in control subjects was $80.0 / 1000$ personyears, which is high compared with that in other reports because control subjects were ascertained through service professionals. We believe the incidence rate of dementia among patients with PD, ie, $112.5 / 1000$ personyears, is accurate.

The present study has advantages, but also limitations. We believe ascertainment of the vast majority of cases of PD in the community was likely. ${ }^{32}$ This is the only study in which a full neuropsychological battery rather than a screening mental status examination was used to determine whether DSM-III-R criteria for dementia were met. The use of a neuropsychological battery rather than a screening test minimizes diagnostic error in the determination of dementia. Functional impairment secondary to cognitive rather than motor impairment was necessary to diagnose a subject as having dementia.

Although we are confident that patients met research criteria for $\mathrm{PD}^{8-10}$ and DSM-III-R criteria for dementia, pathological confirmation of the cause of the dementia is lacking. Controversy over the importance of Lewy body disease as an explanation of dementia in PD may have different implications for progression. It is unclear whether extrapyramidal signs, HDRS scores, or any other variables predict the development of dementia in patients with Lewy body disease.

Patients with PD were taking a variety of medications at the time of the baseline examination. Levodopa, dopamine agonist, and anticholinergic use was not significantly different in those patients who remained nondemented as compared with that in those who became demented. Because no patient was demented when the examinations were initially performed, it is unlikely that medications were adjusted or discontinued for cognitive impairment, thereby resulting in a higher EPS.

The presence of extrapyramidal signs possibly mediated by the degeneration of dopaminergic pathways predicts the development of dementia in patients with PD. Depressive symptoms, too, may serve as a marker of low metabolic reserves in the prefrontal cortex. Although the relative contribution of various neurotransmitter deficiencies is unknown, severity of extrapyramidal signs and possibly depressive symptoms in patients with PD may predict the development of dementia. Patients with PD, especially those with more severe extrapyramidal signs, have nearly twice the risk for the development of dementia as compared with that in control subjects.

Accepted for publication June 13, 1994.

This work was supported by grants AG072.32, AG08702, RR00645, and AG10963 from the National Institutes of Health, Bethesda, Md, and by a grant from the Parkinson's Disease Foundation, New York.

Reprint requests to Gertrude H. Sergievsky Center, 630 W 168th St, New York, NY 10032 (Dr Marder).

\section{REFERENCES}

1. Mayeux $R$, Stern $Y$, Rosenstein $R$, et al. The prevalence of dementia in idiopathic Parkinson's disease. Arch Neurol. 1988;45:260-262.

2. Marder K, Flood P, Cote L, Mayeux R. A pilot study of risk factors for dementia in Parkinson's disease. Mov Disord. 1990;5:156-161.

3. Salganik 1, Korczyn A. Risk factors for dementia in Parkinson's disease. Adv Neurol. 1990;53:343-347.

4. Biggins $C$, Boyd J, Harrop F, et al. A controlled, longitudinal study of dementia in Parkinson's disease. J Neurol Neurosurg Psychiatry. 1992;55:566-571.

5. Ebmeier KP, Calder SA, Crawford JR, Stewart L, Besson JA, Mutch WJ. Clinical features predicting dementia in idiopathic Parkinson's disease: a follow-up study. Neurology. 1990;40:1222-1224.

6. Starkstein S, Mayberg H, Leiguarda R, Preziosi T, Robinson R. A prospective longitudinal study of depression, cognitive decline, and physical impairments in patients with Parkinson's disease. J Neurol Neurosurg Psychiatry. 1992;55: 377-382.

7. Stern Y, Marder K, Cote L, Mayeux R. Antecedent clinical factors associated with dementia in Parkinson's disease. Neurology. 1993;43:1690-1692.

8. Ward CD, Gibb WR. Research diagnostic criteria for Parkinson's disease. In: Streiffler MB, Korczyn AD, Melamed E, Youdim MB, eds. Parkinson's Disease: Anatomy, Pathology, and Therapy. New York, NY: Raven Press; 1990:245249.

9. Hughes AJ, Daniel SE, Kilford L, Lees AJ. Accuracy of clinical diagnosis of idiopathic Parkinson's disease: a clinico-pathological study of 100 cases. $J$ Neurol Neurosurg Psychiatry. 1992;55:181-184.

10. Hughes AJ, Ben-Schlomo Y, Daniel SE, Lees AJ. What features improve the 
accuracy of clinical diagnosis in Parkinson's disease: a clinicopathologic study. Neurology. 1992;42:1142-1146.

11. Mayeux R, Stern Y, Spanton S. Heterogeneity of dementia of the Alzheimer type: evidence of subgroups. Neurology. 1985;35:453-461.

12. Stern MB. The clinical characteristics of Parkinson's disease and parkinsonian syndromes: diagnosis and assessment. In: Stern MB, Hurtig HI, eds. The Comprehensive Management of Parkinson's Disease. New York, NY: PMA Publishing Corp; 1978:34-39.

13. Richards M, Marder K, Cote L, Mayeux R. Interrater reliability and factor structure of the Unified Parkinson's Disease Rating Scale Motor Examination. Mov Disord. 1994:9:89-91.

14. American Psychiatric Association. Diagnostic and Statistical Manual of Mental Disorders, Revised Third Edition. Washington, DC: American Psychiatric Association; 1987

15. Stern Y, Andrews H, Pittman J, et al. Diagnosis of dementia in a heterogeneous population: development of a neuropsychological paradigm-based diagnosis of dementia and quantified correction for the effects of education. Arch Neurol. 1992;49:453-460.

16. Schwab JF, England AC. Projection technique for evaluating surgery in Parkinson's disease. In: Gillinghan FS, Donaldson MN, eds. Third Symposium on Parkinson's Disease. Edinburgh, Scotland: E \& S Livingstone; 1969:152-157.

17. Williams JBW. A structured interview guide for the Hamilton Depression Rating Scale. Arch Gen Psychiatry. 1988:45:742-747.

18. Mahoney FI, Barthel DW. Functional evaluation: the Barthel index. Md Med J. 1965;14:61-65.

19. Blessed G, Tomlinson BE, Roth $M$. The association between quantitative measures of dementia and of senile changes in the cerebral grey matter of elderly subjects. Br J Psychol. 1968;225:797-811.

20. Richards $M$, Stern $Y$, Mayeux R. Subtle extrapyramidal signs can predict the development of dementia in elderly individuals. Neurology. 1993;43:21842188
21. Mortimer JA, Pirozzolo FJ, Hansch EC, Webster DD. Relationship of motor symptoms to intellectual deficits in Parkinson's disease. Neurology. 1982;32: 133-137.

22. Rinne JO, Rummukainen J, Paljarvi L, Rinne UK. Dementia in Parkinson's disease is related to neuronal loss in the medial substantia nigra. Ann Neurol $1989 ; 26: 47-50$

23. Pizzolato $\mathrm{G}$, Dam M, Borsato N, et al. [99mTC]-HM-PAO SPECT in Parkinson's disease. J Cereb Blood Flow Metab. 1988;8(suppl):S101-S108

24. Spampinato U, Habert MO, Mas JL, et al. (99mTC)-HM-PAO SPECT and cognitive impairment in Parkinson's disease: a comparison with dementia of the Alzheimer's type. J Neurol Neurosurg Psychiatry. 1991;54:787-792.

25. Sawada H, Fukashi U, Masakuni K, et al. SPECT findings in Parkinson's disease associated with dementia. J Neurol Neurosurg Psychiatry. 1992;55;960-963.

26. Cummings J. Depression and Parkinson's disease: a review. Am J Psychiatry. 1992;149:443-454.

27. Torack RM, Morris JC. The association of ventral tegmental area histopathology with adult dementia. Arch Neurol. 1988;45:497-501.

28. Mayberg HS, Starkstein S, Sadzot B, et al. Selective inferior frontal lobe hypometabolism in depressed patients with Parkinson's disease. Ann Neurol. 1990; 28:57-64

29. Mayeux R, Stern Y, Williams JBW, Cote L, Franz A, Dyrenfurth I. Clinical and biochemical features of depression in Parkinson's disease. Am J Psychiatry. 1986;143:756-759.

30. Kostic VS, Djuricic BM, Covickovic-Sternic N, Bumbasirevic L, Nikolic M, Mrsulja B. Depression and Parkinson's disease: possible role of serotonergic mechanisms. J Neurol. 1987;2:94-96.

31. Sano M, Stern Y, Williams J, Cote L, Rosenstein R, Mayeux R. Coexisting dementia and depression in Parkinson's disease. Arch Neurol. 1989;46:1284-1286.

32. Mayeux R, Denaro J, Hemenegildo $\mathrm{N}$, et al. A population-based investigation of Parkinson's disease with and without dementia: relationship to age and gender. Arch Neurol. 1992:49:492-500.

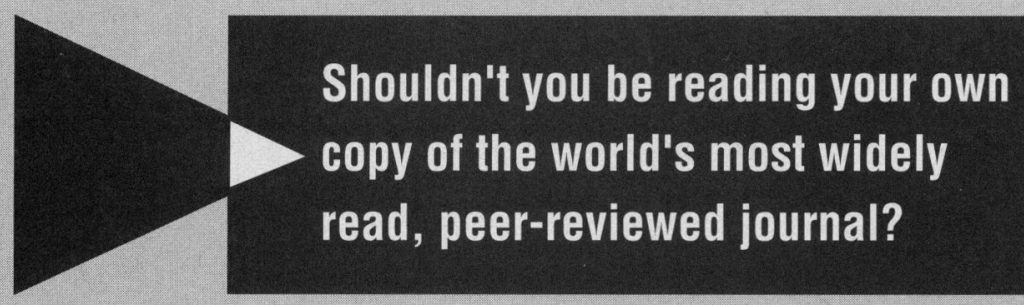

Subscribe to JAMA: The Journal of the American Medical Association today!
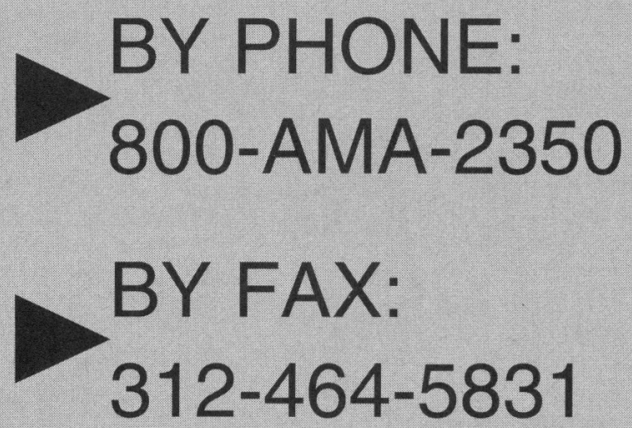

BY MAIL:

Q Enter my one-year subscription to JAMA (48 issues) for $\$ 120$

Name

$\mathrm{MD} / \mathrm{DO} \mathrm{O}$ Other

Please Specify

Address

City/State/Zip

O Check enclosed payable to the American Medical Association (for mail orders).

Please charge my

O VISA O American Express O MasterCard Card No.

Exp. Date

Signature

By Mail: Subscriber Services American Medical Association P.0. Box 10945 Chicago, IL 60610

Washington, DC residents add $6 \%$ sales tax. Canad residents add $7 \%$ GST. Institution rate is $\$ 160$. Individual rate does not apply if payment is made through an institution. Please add $\$ 40$ (institutions add $\$ 60$ ) for orders delivered outside the USA. Rate subject to change. 